\title{
Transport de l'humidité dans des plaques minces stratifiées
}

\author{
Lahsen Ouissaden $^{1}$, Ahmed Lekhder $^{2}$, Hélène Dumontet $^{3, a}$ et Abelwahed Benhamida ${ }^{3}$ \\ 1 Département de Physique, Centre Pédagogique Régional (CPR), Souissi, BP 6210 Rabat, Maroc \\ 2 Laboratoire de Mécanique et des Matériaux, Faculté des Sciences, Agdal, BP 1014 Rabat, Maroc \\ 3 Laboratoire de Modélisation, Matériaux et Structures, Université Paris VI, CC 161, 4 place Jussieu, 75252 Paris Cedex 05, \\ France
}

Reçu le 27 janvier 2004, accepté le 21 avril 2005

\begin{abstract}
Résumé - Une modélisation du transport de l'humidité dans des plaques minces stratifiées, qui intègre le phénomène de diffusion préférentielle le long des interfaces entre les plis, est proposée dans ce travail. Une loi d'interface surfacique, asymptotiquement équivalente à une très fine couche de forte diffusivité, est tout d'abord établie par des techniques asymptotiques, puis introduite au niveau des intercouches d'une plaque mince stratifiée. Des développements asymptotiques selon l'épaisseur de la plaque permettent alors de proposer un modèle bidimensionnel équivalent pour le comportement hygroscopique de plaque lamifiée dans le cas où les faces supérieure et inférieure de la plaque sont hygroscopiquement isolées. Des applications numériques viennent enfin valider et illustrer ces modèles asymptotiques.
\end{abstract}

Mots clés : Transport de l'humidité / plaques minces stratifiées / diffusion interfaciale / techniques asymptotiques

\begin{abstract}
Moisture transport in thin stratified plates. In this paper a modelling of the transport of the moisture in thin stratified plates, which integrates the phenomenon of preferential diffusion along the interfaces between the plies, is proposed. One thus establishes by asymptotic techniques a surfacic law, asymptotically equivalent to a thin layer with a strong diffusivity and then, introduces it between the interlayers of a laminated plate. Asymptotic developments according to the thickness of the plate make it possible to propose a two-dimensional equivalent model for the hygroscopic behavior of laminated plate in the case of insulated upper and lower surfaces. Various numerical applications allow to validate and illustrate these asymptotic models.
\end{abstract}

Key words: Transport of moisture / thin stratified plates / interfacial diffusion / asymptotic methods

\section{Introduction}

Les matériaux composites, de par leurs propriétés de flottabilité, de résistance à la corrosion et de grande souplesse de conception, occupent une place importante dans la fabrication de structures navales et sous-marines. Cette utilisation en milieu marin particulièrement hostile, à des profondeurs pouvant atteindre 6000 mètres, requiert une bonne maîtrise des phénomènes d'absorption d'eau, qui sont souvent rendus responsables de dégradations des matériaux. Typiquement, des expositions prolongées à un milieu humide peuvent conduire à un gonflement différentiel de la matrice qui engendre l'apparition de contraintes résiduelles ou encore une dégradation

\footnotetext{
a Auteur correspondant : dumontet@ccr.jussieu.fr
}

physico-chimique de la matrice ou une hydrolyse qui modifient son comportement et entraînent un vieillissement prématuré, une modification du seuil de plasticité ou bien encore à une dégradation de la liaison entre les constituants. Ces dégradations peuvent alors conduire à une accélération du processus de propagation et, à terme, à la ruine du matériau. De tels phénomènes ont été largement observés expérimentalement, on pourra consulter par exemple les travaux de G.S. Springer [1], B. Dewimille [2], R. Delasi et J.B. Whiteside [3].

Plusieurs modèles de diffusion ont été proposés pour analyser le phénomène d'absorption d'eau dans les matériaux composites A.C.H. Shen [4], A. Loos et G.S. Springer [5], G.S Springer et S.W. Tsai [6], J.P. Favre et J.N. Dewas [7], J. Crank [8], les plus classiques étant les modèles de Fick et de Langmuir. Le modèle fickien 
de diffusion est formé à partir de la loi de Fourier par analogie entre les phénomènes de transfert de chaleur et de transfert de masse. Le modèle de Langmuir est lui au contraire un modèle de diffusion à deux phases, une phase étant libre de diffuser et l'autre étant piégée. Autant ces modèles sont bien adaptés pour décrire le phénomène d'absorption d'humidité dans les milieux homogènes, autant ils peuvent apparaître comme relativement insuffisants pour décrire le phénomène de diffusion dans les matériaux composites. Ils prédisent en effet une prise de poids en eau du composite moindre que celle effectivement observée expérimentalement, R. Baizeau [9], P. Bonniau et A.R. Bunsell [10], B. Dewimille et A.R. Bunsell [11]. Pour expliquer cette insuffisance, il est couramment avancé que les modèles ne tiennent pas compte du rôle préférentiel que peuvent jouer les interfaces entre les constituants des composites dans l'accélération du processus d'absorption. Des observations expérimentales ont en effet mis en évidence ce rôle préférentiel, P. Kanouté [12], A. Loos et G.S. Springer [5], J.P. Favre [13], C. Schutte [14].

Quelques travaux ont été consacrés à la modélisation du comportement fortement diffusif des interfaces dans certains types de composites, comme des composites à fibres unidirectionnelles, A. Benhamida et al. [15], N. Lebris [16], P. Kanouté [12] ou des mousses syntactiques, A. Benhamida et al. [17], A. Lekhder [18]. Dans ces travaux, cette modélisation interfaciale a été introduite dans une approche d'homogénéisation périodique pour caractériser le comportement hygrométrique équivalent des composites en présence d'interfaces diffusives ou piégeantes. Des comparaisons avec des études expérimentales avaient alors permis de valider les modèles proposés et d'identifier les caractéristiques diffusives et piégeantes des interfaces.

C'est en s'inspirant de ces approches que nous proposons dans ce travail une modélisation du phénomène de diffusion de l'humidité dans des plaques minces stratifiées. Une loi interfaciale diffusive surfacique est tout d'abord proposée à la section 2 pour simuler le comportement d'une interphase plane entre deux couches du stratifié, très mince et fortement diffusive. Il est procédé à une adimensionnalisation du problème de transmission de l'humidité à travers cette interphase et deux petits paramètres sont introduits, le rapport de l'épaisseur de l'interphase et de sa dimension transversale et le rapport de diffusivité d'un pli et de l'interphase. Le comportement limite est établi formellement par des techniques asymptotiques, lorsque les deux petits paramètres, supposés du même ordre, tendent vers zéro. La section 3 est ensuite consacrée au comportement diffusif équivalent d'une plaque mince stratifiée, chacune des interfaces présentant le comportement hygrométrique précédemment établi, les faces supérieure et inférieure de la plaque étant par ailleurs hygroscopiquement isolées. La technique des développements asymptotiques est cette fois mise en ouvre avec comme petit paramètre le rapport de l'épaisseur de la plaque et de sa dimension transversale. Le modèle limite équivalent est bidimensionnel et défini sur le plan moyen de la plaque. La mise en œuvre numérique de ces comportements limites est enfin présentée à la section 4 et différentes applications permettent de s'assurer de leur validité.

\section{Loi de diffusion interfaciale}

\subsection{Formulation du problème de transmission dans l'interphase}

Considérons le problème de transmission de l'humidité à travers une interphase située entre deux couches d'un stratifié. On convient de noter $\boldsymbol{e}_{3}$ la direction d'empilement des couches, e l'épaisseur de l'interphase selon la direction $\boldsymbol{e}_{3}, L$ sa dimension selon les directions transverses $\boldsymbol{e}_{1}$ et $\boldsymbol{e}_{2}$. On désigne par $\Omega=[0, L] \times[0, L] \times[0, e]$ le domaine occupé par l'interphase.

La concentration en eau en un point $x=\left(x_{1}, x_{2}, x_{3}\right)$ de l'interphase et à l'instant $t$ est notée $c(x, t)$ et représente la masse d'eau par unité de volume contenue au point $x$ à l'instant $t$. Le vecteur flux d'humidité par unité d'aire, noté $\boldsymbol{q}(x, t)$, est relié à tout instant $t$ au gradient de concentration en eau par la loi de Fick, J. Crank [8] :

$$
\boldsymbol{q}(x, t)=-D \nabla c(x, t), \operatorname{dans} \Omega
$$

où $\boldsymbol{D}$ désigne le tenseur de diffusion hygrométrique et $\nabla$ l'opérateur gradient en $x$ d'une fonction scalaire.

L'équation locale de conservation de la masse dans l'interphase s'écrit en tout point $x$ et à tout instant $t$ :

$$
\frac{\partial c}{\partial t}(x, t)+\nabla \cdot \boldsymbol{q}(x, t)=0, \text { dans } \Omega
$$

où $\nabla$. désigne l'opérateur divergence d'un vecteur.

On désigne par $\Gamma^{+}$l'interface d'équation $x_{3}=e$ entre la couche supérieure du stratifié et l'interphase et par $\Gamma^{-}$l'interface d'équation $x_{3}=0$ entre la couche inférieure et l'interphase. Notons enfin par $c^{+}$(respectivement $c^{-}$) la valeur de la concentration en eau dans la couche supérieure (respectivement inférieure) prise sur l'interface $\Gamma^{+}$(respectivement $\Gamma^{-}$) et par $g^{+}$(respectivement $g^{-}$) la valeur du flux normal d'humidité dans la couche supérieure (respectivement inférieure) prise sur l'interface $\Gamma^{+}$(respectivement $\left.\Gamma^{-}\right)$.

L'interphase est supposée très mince devant ses dimensions transversales. Dans la perspective de la section 3 où cette interphase sera introduite entre les couches d'une plaque mince stratifiée d'épaisseur $\epsilon$, on convient de noter :

$$
e=\eta \epsilon, \quad \epsilon=\xi L, \quad \text { soit donc } \quad \frac{e}{L}=\eta \xi
$$

et on a :

$$
\eta<<1 \quad \text { et } \quad \xi \text { fixé à ce stade }
$$

Nous supposons de plus qu'elle présente un comportement diffusif, homogène, isotrope, de sorte que le tenseur 
$\boldsymbol{D}$ est sphérique, de la forme $\boldsymbol{D}=D \boldsymbol{I}, \boldsymbol{I}$ désignant le tenseur identité d'ordre 2. Les plis adjacents sont également supposés homogènes et isotropes.

Par ailleurs, en s'appuyant sur des observations expérimentales, C. Schutte [14], P. Kanouté [12], J.P. Favre [13], l'interphase est supposée fortement diffusive par rapport aux comportements des plis adjacents qui sont eux du même ordre de diffusivité, de sorte que l'on a :

$$
\delta=\frac{D^{+}}{D}<<1
$$

où $D^{+}$représente la diffusivité de la couche supérieure.

\subsection{Comportement asymptotique de l'interphase}

Proposition 1 : La loi d'interface suivante :

$$
[[c]]=0, \quad[[\boldsymbol{q} \cdot \boldsymbol{n}]]=\epsilon \tilde{D} \Delta_{T} c=\epsilon \tilde{D}\left(\frac{\partial^{2} c}{\partial x_{1}^{2}}+\frac{\partial^{2} c}{\partial x_{2}^{2}}\right)
$$

est asymptotiquement équivalente au problème de transmission de l'humidité à travers une interphase d'épaisseur $\eta \epsilon$ et de diffusivité $\tilde{D} / \delta$, lorsque les petits paramètres $\delta$ et $\eta$ sont du même ordre et tendent vers zéro simultanément, $\epsilon$ et $\tilde{D}$ étant fixés.

Dans ces relations, les crochets $[[f]]$ désignent le saut de la fonction $f$ à l'interface, soit $[[f]]=f^{+}-f^{-}$, où les indices + et - se rapportent respectivement à la couche supérieure et la couche inférieure, $\boldsymbol{n}$ désignant la normale unitaire extérieure à la couche supérieure.

Preuve : Nous nous proposons d'établir formellement ce résultat de convergence à l'aide de la technique des développements asymptotiques. Au préalable, nous procédons à une adimensionnalisation des équations du problème de transmission. On convient d'indicer par $*$ les nouvelles variables adimensionnées et par $r$ les constantes caractéristiques de référence. En retenant comme caractéristiques de référence pour la diffusivité et le temps celles de la couche supérieure, on a ainsi, compte tenu de (5), les relations suivantes :

$$
\begin{aligned}
x_{\alpha}=L x_{\alpha}^{*}, \quad \alpha=(1,2), \quad x_{3} & =\eta \epsilon x_{3}^{*}, \quad t=\frac{L^{2}}{D^{+}} t^{*} \\
c & =c_{r} c^{*}, \quad D=\frac{D^{+}}{\delta} D^{*}
\end{aligned}
$$

Le problème de transmission s'écrit alors sous la forme d'un problème adimensionné $P^{*}$ posé sur le domaine
$\Omega^{*}=[0,1] \times[0,1] \times[0,1]$, comportant deux petits paramètres $\eta$ et $\delta, \xi$ étant fixé à ce stade :

$$
\begin{gathered}
\frac{\partial c^{*}}{\partial t^{*}}\left(x^{*}, t^{*}\right)-\frac{D^{*}}{\delta} \frac{\partial^{2} c^{*}}{\partial x_{\alpha}^{*} \partial x_{\alpha}^{*}}\left(x^{*}, t^{*}\right) \\
-\frac{D^{*}}{\delta \eta^{2} \xi^{2}} \frac{\partial^{2} c^{*}}{\partial x_{3}^{* 2}}\left(x^{*}, t^{*}\right)=0, \text { dans } \Omega^{*}, \\
c^{*}\left(x_{1}^{*}, x_{2}^{*}, x_{3}^{*}=1, t^{*}\right)=c_{+}^{*}=\frac{c^{+}}{c_{r}}, \\
c^{*}\left(x_{1}^{*}, x_{2}^{*}, x_{3}^{*}=0, t^{*}\right)=c_{-}^{*}=\frac{c^{-}}{c_{r}}, \\
\boldsymbol{q}^{*}\left(x_{1}^{*}, x_{2}^{*}, x_{3}^{*}=1, t^{*}\right) \cdot \boldsymbol{n}= \\
\quad-\frac{D^{*}}{\delta \eta \xi} \frac{\partial c^{*}}{\partial x_{3}^{*}}\left(x_{1}^{*}, x_{2}^{*}, x_{3}^{*}=1, t^{*}\right)=g_{+}^{*}=\frac{L}{D^{+} c_{r}} g^{+}, \\
\boldsymbol{q}^{*}\left(x_{1}^{*}, x_{2}^{*}, x_{3}^{*}=0, t^{*}\right) \cdot \boldsymbol{n}= \\
\frac{D^{*}}{\delta \eta \xi} \frac{\partial c^{*}}{\partial x_{3}^{*}}\left(x_{1}^{*}, x_{2}^{*}, x_{3}^{*}=0, t^{*}\right)=g_{-}^{*}=\frac{L}{D^{+} c_{r}} g^{-}
\end{gathered}
$$

Ce système d'équations demande à être complété par une condition initiale, ainsi que des conditions aux limites sur les bords latéraux de l'interphase, qu'il ne nous est pas nécessaire de préciser à ce stade.

La concentration $c^{*}$ dans l'interphase, solution du problème de transmission $P^{*}$, ainsi que les concentrations $c_{+}^{*}$ et $c_{-}^{*}$ dans les plis supérieur et inférieur et les flux $g_{+}^{*}$ et $g_{-}^{*}$ sont recherchés à tout instant $t^{*}$ sous la forme des développements suivants :

$$
\begin{aligned}
& c^{*}\left(x_{1}^{*}, x_{2}^{*}, x_{3}^{*}, t^{*}\right)=c^{0}\left(x_{1}^{*}, x_{2}^{*}, x_{3}^{*}, t^{*}\right) \\
& \quad+\eta c^{1}\left(x_{1}^{*}, x_{2}^{*}, x_{3}^{*}, t^{*}\right)+\eta^{2} c^{2}\left(x_{1}^{*}, x_{2}^{*}, x_{3}^{*}, t^{*}\right)+\cdots,
\end{aligned}
$$

$$
\begin{aligned}
& c_{ \pm}^{*}\left(x_{1}^{*}, x_{2}^{*}, x_{3}^{*}, t^{*}\right)=c_{ \pm}^{0}\left(x_{1}^{*}, x_{2}^{*}, x_{3}^{*}, t^{*}\right) \\
& +\eta c_{ \pm}^{1}\left(x_{1}^{*}, x_{2}^{*}, x_{3}^{*}, t^{*}\right)+\eta^{2} c_{ \pm}^{+2}\left(x_{1}^{*}, x_{2}^{*}, x_{3}^{*}, t^{*}\right)+\cdots,
\end{aligned}
$$

$$
\begin{aligned}
& g_{ \pm}^{*}\left(x_{1}^{*}, x_{2}^{*}, x_{3}^{*}, t^{*}\right)=g_{ \pm}^{0}\left(x_{1}^{*}, x_{2}^{*}, x_{3}^{*}, t^{*}\right) \\
& \quad+\eta g_{ \pm}^{1}\left(x_{1}^{*}, x_{2}^{*}, x_{3}^{*}, t^{*}\right)+\eta^{2} g_{ \pm}^{2}\left(x_{1}^{*}, x_{2}^{*}, x_{3}^{*}, t^{*}\right)+\cdots
\end{aligned}
$$

Substituons ces développements dans les équations du problème $P^{*}$ et identifions les problèmes aux différents ordres. En supposant $\delta=O(\eta)$ et $\xi$ fixé, on obtient les problèmes suivants :

Ordre $\eta^{-3}$ :

$$
\begin{gathered}
D^{*} \frac{\partial^{2} c^{0}}{\partial x_{3}^{* 2}}\left(x_{1}^{*}, x_{2}^{*}, x_{3}^{*}, t^{*}\right)=0, \text { dans } \Omega^{*}, \\
c^{0}\left(x_{1}^{*}, x_{2}^{*}, x_{3}^{*}=1, t^{*}\right)=c_{+}^{0}\left(x_{1}^{*}, x_{2}^{*}, x_{3}^{*}=1, t^{*}\right), \\
c^{0}\left(x_{1}^{*}, x_{2}^{*}, x_{3}^{*}=0, t^{*}\right)=c_{-}^{0}\left(x_{1}^{*}, x_{2}^{*}, x_{3}^{*}=0, t^{*}\right), \\
D^{*} \frac{\partial c^{0}}{\partial x_{3}^{*}}\left(x_{1}^{*}, x_{2}^{*}, x_{3}^{*}=1, t^{*}\right)= \\
D^{*} \frac{\partial c^{0}}{\partial x_{3}^{*}}\left(x_{1}^{*}, x_{2}^{*}, x_{3}^{*}=0, t^{*}\right)=0
\end{gathered}
$$


Les variables $x_{1}^{*}, x_{2}^{*}$ et $t^{*}$ jouant ici le rôle de paramètres, la solution de ce problème est de manière évidente :

$$
c^{0}\left(x_{1}^{*}, x_{2}^{*}, x_{3}^{*}, t^{*}\right)=c^{0}\left(x_{1}^{*}, x_{2}^{*}, t^{*}\right)
$$

De sorte que d'après (17) et (18), on a :

$$
c_{-}^{0}\left(x_{1}^{*}, x_{2}^{*}, x_{3}^{*}=0, t^{*}\right)=c_{+}^{0}\left(x_{1}^{*}, x_{2}^{*}, x_{3}^{*}=1, t^{*}\right)
$$

soit donc, continuité de la concentration en eau à l'interface entre les plis.

Ordre $\eta^{-2}$ :

$$
\begin{gathered}
D^{*} \frac{\partial^{2} c^{1}}{\partial x_{3}^{* 2}}\left(x_{1}^{*}, x_{2}^{*}, x_{3}^{*}, t^{*}\right)=0, \text { dans } \Omega^{*}, \\
c^{1}\left(x_{1}^{*}, x_{2}^{*}, x_{3}^{*}=1, t^{*}\right)=c_{+}^{1}\left(x_{1}^{*}, x_{2}^{*}, x_{3}^{*}=1, t^{*}\right), \\
c^{1}\left(x_{1}^{*}, x_{2}^{*}, x_{3}^{*}=0, t^{*}\right)=c_{-}^{1}\left(x_{1}^{*}, x_{2}^{*}, x_{3}^{*}=0, t^{*}\right), \\
D^{*} \frac{\partial c^{1}}{\partial x_{3}^{*}}\left(x_{1}^{*}, x_{2}^{*}, x_{3}^{*}=1, t^{*}\right)= \\
D^{*} \frac{\partial c^{1}}{\partial x_{3}^{*}}\left(x_{1}^{*}, x_{2}^{*}, x_{3}^{*}=0, t^{*}\right)=0
\end{gathered}
$$

ce qui conduit là encore à la solution :

$$
c^{1}\left(x_{1}^{*}, x_{2}^{*}, x_{3}^{*}, t^{*}\right)=c^{1}\left(x_{1}^{*}, x_{2}^{*}, t^{*}\right) .
$$

Ordre $\eta^{-1}$ :

$$
\begin{gathered}
\frac{D^{*}}{\xi^{2}} \frac{\partial^{2} c^{2}}{\partial x_{3}^{* 2}}\left(x_{1}^{*}, x_{2}^{*}, x_{3}^{*}, t^{*}\right)= \\
\quad-D^{*}\left(\frac{\partial^{2} c^{0}}{\partial x_{1}^{* 2}}+\frac{\partial^{2} c^{0}}{\partial x_{2}^{* 2}}\right)\left(x_{1}^{*}, x_{2}^{*}, t^{*}\right), \operatorname{dans} \Omega^{*} \\
c^{2}\left(x_{1}^{*}, x_{2}^{*}, x_{3}^{*}=1, t^{*}\right)=c_{+}^{2}\left(x_{1}^{*}, x_{2}^{*}, x_{3}^{*}=1, t^{*}\right) \\
c^{2}\left(x_{1}^{*}, x_{2}^{*}, x_{3}^{*}=0, t^{*}\right)=c_{-}^{2}\left(x_{1}^{*}, x_{2}^{*}, x_{3}^{*}=0, t^{*}\right) \\
-\frac{D^{*}}{\xi} \frac{\partial c^{2}}{\partial x_{3}^{*}}\left(x_{1}^{*}, x_{2}^{*}, x_{3}^{*}=1, t^{*}\right)=g_{+}^{0}\left(x_{1}^{*}, x_{2}^{*}, x_{3}^{*}=1, t^{*}\right) \\
\frac{D^{*}}{\xi} \frac{\partial c^{2}}{\partial x_{3}^{*}}\left(x_{1}^{*}, x_{2}^{*}, x_{3}^{*}=0, t^{*}\right)=g_{-}^{0}\left(x_{1}^{*}, x_{2}^{*}, x_{3}^{*}=0, t^{*}\right)
\end{gathered}
$$

Pour $\left(x_{1}^{*}, x_{2}^{*}, t^{*}\right)$ fixés, on vérifie aisément que ce problème est bien posé et admet une solution $c^{2}\left(x_{3}^{*}\right)$ unique. En intégrant l'équation $(27)$ en $x_{3}^{*}$ entre 0 et 1 et en exploitant les conditions de transmission (30), (31), on obtient la relation :

$$
\left[\left[g^{0}\right]\right]=D^{*} \xi\left(\frac{\partial^{2} c^{0}}{\partial x_{1}^{* 2}}\left(x_{1}^{*}, x_{2}^{*}, t^{*}\right)+\frac{\partial^{2} c^{0}}{\partial x_{2}^{* 2}}\left(x_{1}^{*}, x_{2}^{*}, t^{*}\right)\right)
$$

En revenant maintenant aux variables dimensionnées, on obtient le résultat annoncé.

Des lois de ce type ont été également rencontrées dans des problèmes de transmissions dans des couches minces en thermique, H.S. Carslaw et J.C. Jaeger [19],
H.P. Huy et E. Sanchez-Palencia [20], ainsi qu'en électromagnétisme, A. Bendali [21]. Le cas $\tilde{D}=0$ correspond à une interphase non diffusive, on retrouve alors les conditions de transmission classiques entre deux constituants parfaitement collés.

Nous nous intéressons maintenant à la résolution du problème de diffusion de l'humidité dans une plaque mince stratifiée dont les interfaces entre les plis fortement diffusives sont modélisées par la loi surfacique (6). Les faces supérieure et inférieure de la plaque sont supposées isolées hygroscopiquement. Compte tenu de la faible épaisseur de la plaque, nous pouvons établir un modèle bidimensionnel de transport de l'humidité formulé sur le plan moyen de la plaque.

\section{Un modèle bidimensionnel de diffusion de l'humidité dans une plaque mince stratifiée}

\section{1 Équations du problème tridimensionnel}

Considérons une plaque stratifiée d'épaisseur constante $\epsilon$. Un point de la plaque est repéré par ses coordonnées cartésiennes $x=\left(x_{1}, x_{2}, x_{3}\right)$ dans le repère $\left(0, \boldsymbol{e}_{1}, \boldsymbol{e}_{2}, \boldsymbol{e}_{3}\right)$ avec $\left(x_{1}, x_{2}\right) \in \omega$, le plan moyen de la plaque et $x_{3} \in[-\epsilon / 2, \epsilon / 2]$. La plaque est supposée mince, c'est-à-dire que son épaisseur $\epsilon$ est petite devant les dimensions caractéristiques $L$ de son plan moyen et stratifiée dans la direction $\boldsymbol{e}_{3}$. Les $N$ plis de la plaque sont constitués de renforts unidirectionnels imprégnés de résine, l'orientation des fibres variant d'un pli à l'autre. On admettra, Y. Chevalier [22], que le comportement diffusif d'un pli est homogène et isotrope transverse dans le repère $\left(0, \boldsymbol{e}_{L}, \boldsymbol{e}_{T}, \boldsymbol{e}_{3}\right)$ où l'indice $L$ désigne la direction longitudinale des renforts et l'indice $T$ la direction transversale. Le tenseur de diffusivité hygroscopique d'un pli $\boldsymbol{D}_{\mathrm{p}}$ dans ce repère est donc de la forme suivante :

$$
\boldsymbol{D}_{\mathrm{p}}=\left(\begin{array}{ccc}
D_{\mathrm{L}} & 0 & 0 \\
0 & D_{\mathrm{T}} & 0 \\
0 & 0 & D_{\mathrm{T}}
\end{array}\right)
$$

où $D_{\mathrm{L}}$ et $D_{\mathrm{T}}$ sont respectivement les coefficients de diffusion longitudinale et transversale du pli. Dans le repère du stratifié, le tenseur de diffusivité d'un pli est donc de la forme :

$$
\boldsymbol{D}_{\mathrm{p}}=\left(\begin{array}{ccc}
D_{11} & D_{12} & 0 \\
D_{12} & D_{22} & 0 \\
0 & 0 & D_{33}
\end{array}\right)
$$

avec :

$$
\begin{gathered}
D_{11}=D_{\mathrm{L}} \cos ^{2} \theta+D_{\mathrm{T}} \sin ^{2} \theta, \\
D_{22}=D_{\mathrm{L}} \sin ^{2} \theta+D_{\mathrm{T}} \cos ^{2} \theta, \\
D_{12}=\left(D_{\mathrm{T}}-D_{\mathrm{L}}\right) \sin \theta \cos \theta, \quad D_{33}=D_{\mathrm{T}}
\end{gathered}
$$

$\theta$ désignant l'angle des fibres avec la direction $\boldsymbol{e}_{1}$. 
Le comportement diffusif de la plaque hétérogène est alors caractérisé par le tenseur de diffusivité $\boldsymbol{D}^{\epsilon}=\boldsymbol{D}^{\epsilon}\left(x_{3}\right)$, constant dans chacun des plis. Les $(N-1)$ interfaces planes entre chaque pli, que l'on désigne par $\gamma_{i}^{\epsilon}$, avec $i=$ $1,2, \cdots, N-1$, jouent un rôle diffusif et sont modélisées par la loi d'interface énoncée à la proposition 1 .

La plaque, initialement sèche, est soumise à une concentration d'humidité $c_{a}$ uniforme sur sa surface latérale $\Gamma$, alors que ses faces supérieure et inférieure $x_{3}= \pm \epsilon / 2$ sont supposées hygroscopiquement isolées. La concentration d'humidité $c^{\epsilon}(x, t)$ et le flux d'humidité $\boldsymbol{q}^{\epsilon}(x, t)$ qui règnent dans la plaque hétérogène sont régis à l'instant $t$ par les équations suivantes :

$$
\begin{aligned}
& \frac{\partial c^{\epsilon}}{\partial t}(x, t)+\nabla \cdot \boldsymbol{q}^{\epsilon}(x, t)=0 \\
&\left.\operatorname{dans} \Omega^{\epsilon}=\omega \times\right]-\epsilon / 2, \epsilon / 2[ \\
& \boldsymbol{q}^{\epsilon}(x, t)=-\boldsymbol{D}^{\epsilon} \nabla c^{\epsilon}(x, t), \quad \operatorname{dans} \Omega^{\epsilon} \\
& {\left[\left[c^{\epsilon}\right]\right]=0, \quad \operatorname{sur} \cup \gamma_{i}^{\epsilon}, } \\
& {\left[\left[\boldsymbol{q}^{\epsilon} \cdot \boldsymbol{n}\right]\right]=\epsilon \tilde{D}\left(\frac{\partial^{2} c^{\epsilon}}{\partial x_{1}^{2}}+\frac{\partial^{2} c^{\epsilon}}{\partial x_{2}^{2}}\right), \quad \text { sur } \cup \gamma_{i}^{\epsilon}, } \\
& \boldsymbol{q}^{\epsilon} \cdot \boldsymbol{e}_{3}=0, \quad \operatorname{sur} x_{3}= \pm \epsilon / 2, \\
& c^{\epsilon}(x, t)=c_{a}(x, t), \quad \operatorname{sur} \Gamma
\end{aligned}
$$

\subsection{Comportement asymptotique de plaque mince}

Le problème tridimensionnel ainsi formulé présente un petit paramètre $\xi=\epsilon / L<<1$. Nous nous intéressons maintenant à son comportement asymptotique lorsque $\xi$ tend vers zéro.

Proposition 2: La concentration $c^{\epsilon}\left(x_{1}, x_{2}, x_{3}, t\right)$ solution du problème hétérogène tridimensionnel $P^{\epsilon}(35)-(40)$ converge, lorsque l'épaisseur de la plaque $\epsilon$ tend vers zéro vers $c^{0}\left(x_{1}, x_{2}, t\right)$ solution du problème homogène $P^{0}$ suivant, posé sur le plan moyen de la plaque :

$$
\frac{\partial c^{0}}{\partial t}\left(x_{1}, x_{2}, t\right)-D_{\alpha \beta}^{\mathrm{h}} \frac{\partial^{2} c^{0}}{\partial x_{\alpha} \partial x_{\beta}}\left(x_{1}, x_{2}, t\right)=0, \text { dans } \omega
$$

où les coefficients de diffusion homogènes équivalents $D_{\alpha \beta}^{\mathrm{h}}$ avec $\alpha, \beta=1,2$ sont donnés par :

$$
D_{\alpha \beta}^{\mathrm{h}}=\frac{1}{\epsilon} \int_{-\epsilon / 2}^{\epsilon / 2} D_{\alpha \beta}^{\epsilon}\left(x_{3}\right) \mathrm{d} x_{3}+(N-1) \tilde{D} \delta_{\alpha \beta}
$$

Preuve : Comme précédemment, on procède tout d'abord à une adimensionnalisation du problème $P^{\epsilon}$ en introduisant des variables sans dimension indicées par * et des quantités caractéristiques constantes indicées par $r$, soit :

$$
\begin{array}{r}
x_{\alpha}=L x_{\alpha}^{*}, \alpha=(1,2), \quad x_{3}=\epsilon x_{3}^{*}, \quad t=t_{r} t^{*}, \\
c^{\epsilon}=c_{r} c^{*}, \quad D^{\epsilon}=D_{r} D^{*}
\end{array}
$$

En prenant comme temps caractéristique $t_{r}=L^{2} / D_{r}$ avec comme diffusivité caractéristique celle d'un pli et compte tenu de la forme du tenseur de diffusité avec $D_{\alpha 3}=0$, l'équation de conservation adimensionnée s'écrit :

$$
\begin{aligned}
\frac{\partial c^{*}}{\partial t^{*}}\left(x_{1}^{*}, x_{2}^{*}, x_{3}^{*}, t^{*}\right) & -D_{\alpha \beta}^{*} \frac{\partial^{2} c^{*}}{\partial x_{\alpha}^{*} \partial x_{\beta}^{*}}\left(x_{1}^{*}, x_{2}^{*}, x_{3}^{*}, t^{*}\right) \\
& -\frac{1}{\xi^{2}} D_{33}^{*} \frac{\partial^{2} c^{*}}{\partial x_{3}^{* 2}}\left(x_{1}^{*}, x_{2}^{*}, x_{3}^{*}, t^{*}\right)=0
\end{aligned}
$$

avec les conditions aux interfaces entre les plis :

$$
\left[\left[c^{*}\right]\right]=0, \quad\left[\left[-D_{33}^{*} \frac{\partial c^{*}}{\partial x_{3}^{*}}\right]\right]=\xi^{2} \frac{\tilde{D}}{D_{r}}\left(\frac{\partial^{2} c^{*}}{\partial x_{1}^{* 2}}+\frac{\partial^{2} c^{*}}{\partial x_{2}^{* 2}}\right),
$$$$
\text { sur } \cup \gamma_{i}^{*}
$$

et les conditions aux limites sur les faces supérieure et inférieure de la plaque :

$$
-D_{33}^{*} \frac{\partial c^{*}}{\partial x_{3}^{*}}\left(x_{1}^{*}, x_{2}^{*}, x_{3}^{*}= \pm 1 / 2, t^{*}\right)=0
$$

Recherchons maintenant formellement la concentration $c^{*}\left(x_{1}^{*}, x_{2}^{*}, x_{3}^{*}, t^{*}\right)$ solution de ce problème sous la forme du développement asymptotique en puissance de $\xi$ suivant :

$$
\begin{aligned}
& c^{*}\left(x_{1}^{*}, x_{2}^{*}, x_{3}^{*}, t^{*}\right)=c^{0}\left(x_{1}^{*}, x_{2}^{*}, x_{3}^{*}, t^{*}\right) \\
& \quad+\xi c^{1}\left(x_{1}^{*}, x_{2}^{*}, x_{3}^{*}, t^{*}\right)+\xi^{2} c^{2}\left(x_{1}^{*}, x_{2}^{*}, x_{3}^{*}, t^{*}\right)+\cdots
\end{aligned}
$$

En reportant ce développement dans les équations tridimensionnelles, nous obtenons, pour $\left(x_{1}^{*}, x_{2}^{*}, t^{*}\right)$ fixés, les problèmes suivants aux différents ordres : Ordre $\xi^{-2}$ :

$$
\begin{gathered}
D_{33}^{*} \frac{\partial^{2} c^{0}}{\partial x_{3}^{* 2}}\left(x_{1}^{*}, x_{2}^{*}, x_{3}^{*}, t^{*}\right)=0, \\
\text { pour } \left.x_{3}^{*} \in\right]-1 / 2,+1 / 2[, \\
{\left[\left[c^{0}\right]\right]=\left[\left[-D_{33}^{*} \frac{\partial c^{0}}{\partial x_{3}^{*}}\right]\right]=0, \text { sur } \cup \gamma_{i}^{*},} \\
-D_{33}^{*} \frac{\partial c^{0}}{\partial x_{3}^{*}}\left(x_{1}^{*}, x_{2}^{*}, x_{3}^{*}= \pm 1 / 2, t^{*}\right)=0
\end{gathered}
$$

On vérifie aisément que la fonction $c^{0}\left(x_{3}^{*}\right)$ est l'unique solution du problème variationnel suivant :

$$
\begin{aligned}
& c^{0}\left(x_{3}^{*}\right) \in \mathcal{W} / R, \quad a\left(c^{0}, v\right)= \\
& \quad \int_{-1 / 2}^{+1 / 2} D_{33}^{*} \frac{\partial c^{0}}{\partial x_{3}^{*}} \frac{\partial v}{\partial x_{3}^{*}} \mathrm{~d} x_{3}^{*}=0, \quad \forall v \in \mathcal{W} / R
\end{aligned}
$$

où $\mathcal{W} / R$ désigne l'espace des fonctions de $H^{1}(]-1 / 2$, $+1 / 2[)$ quotienté par les constantes réelles. La fonction $c^{0}\left(x_{3}^{*}\right)$ constante étant solution de ce problème, on en déduit :

$$
c^{0}\left(x_{1}^{*}, x_{2}^{*}, x_{3}^{*}, t^{*}\right)=c^{0}\left(x_{1}^{*}, x_{2}^{*}, t^{*}\right)
$$

Le problème à l'ordre $\xi^{-1}$ satisfait par $c^{1}$ est analogue et conduit de la même manière à :

$$
c^{1}\left(x_{1}^{*}, x_{2}^{*}, x_{3}^{*}, t^{*}\right)=c^{1}\left(x_{1}^{*}, x_{2}^{*}, t^{*}\right)
$$


Ordre $\xi^{0}$ :

$$
\begin{aligned}
& \frac{\partial c^{0}}{\partial t^{*}}-D_{33}^{*} \frac{\partial^{2} c^{2}}{\partial x_{3}^{* 2}}-D_{\alpha \beta}^{*} \frac{\partial^{2} c^{0}}{\partial x_{\alpha}^{*} \partial x_{\beta}^{*}}=0, \\
&\left.\operatorname{pour} x_{3}^{*} \in\right]-1 / 2,+1 / 2[
\end{aligned}
$$

$$
\begin{gathered}
{\left[\left[c^{2}\right]\right]=0,\left[\left[-D_{33}^{*} \frac{\partial c^{2}}{\partial x_{3}^{*}}\right]\right]=\frac{\tilde{D}}{D_{r}}\left(\frac{\partial^{2} c^{0}}{\partial x_{1}^{* 2}}+\frac{\partial^{2} c^{0}}{\partial x_{2}^{* 2}}\right),} \\
\operatorname{sur} \cup \gamma_{i}^{*} \\
-D_{33}^{*} \frac{\partial c^{2}}{\partial x_{3}^{*}}\left(x_{1}^{*}, x_{2}^{*}, x_{3}^{*}= \pm 1 / 2, t^{*}\right)=0
\end{gathered}
$$

On montre alors que la fonction $c^{2}\left(x_{3}^{*}\right)$ est l'unique solution du problème variationnel suivant :

$$
c^{2}\left(x_{3}^{*}\right) \in \mathcal{W} / R, \quad a\left(c^{2}, v\right)=l(v), \quad \forall v \in \mathcal{W} / R
$$

où la forme bilinéaire est donnée par (51) et la forme linéaire par :

$$
\begin{aligned}
l(v)= & -\left(\int_{-1 / 2}^{+1 / 2} v\left(x_{3}^{*}\right) \mathrm{d} x_{3}^{*}\right) \frac{\partial c^{0}}{\partial t^{*}} \\
& +\left(\int_{-1 / 2}^{+1 / 2} D_{\alpha \beta}^{*}\left(x_{3}^{*}\right) v\left(x_{3}^{*}\right) \mathrm{d} x_{3}^{*}\right) \frac{\partial^{2} c^{0}}{\partial x_{\alpha}^{*} \partial x_{\beta}^{*}} \\
& +\frac{\tilde{D}}{D_{r}}\left(\frac{\partial^{2} c^{0}}{\partial x_{1}^{* 2}}+\frac{\partial^{2} c^{0}}{\partial x_{2}^{* 2}}\right) \sum_{i=1}^{N-1} v\left(x_{3}^{*}\right)_{\mid \gamma_{i}^{*}}
\end{aligned}
$$

Ce problème admet une unique solution sous la condition nécessaire $l(\phi)=0$ pour toute fonction $\phi\left(x_{3}\right)$ constante, c'est-à-dire si :

$$
\frac{\partial c^{0}}{\partial t^{*}}-D_{\alpha \beta}^{* \mathrm{~h}} \frac{\partial^{2} c^{0}}{\partial x_{\alpha}^{*} \partial x_{\beta}^{*}}=0, \text { dans } \omega
$$

avec

$$
D_{\alpha \beta}^{* \mathrm{~h}}=\int_{-1 / 2}^{+1 / 2} D_{\alpha \beta}^{*}\left(x_{3}^{*}\right) \mathrm{d} x_{3}^{*}+(N-1) \frac{\tilde{D}}{D_{\mathrm{r}}} \delta_{\alpha \beta}
$$

En revenant aux grandeurs dimensionnées, on retrouve l'équation bidimensionnelle posée sur le plan moyen annoncée.

Des modèles classiques de plaque en élasticité ont été retrouvés et justifiés de cette manière par des méthodes asymptotiques analogues, Ph. Destuynder [23], D. Caillerie [24] ou encore E. Sanchez-Palencia [25].

Il convient de noter que ce problème bidimensionnel équivalent n'a pu être établi que sous l'hypothèse de faces inférieure et supérieure hygroscopiquement isolées. La formulation reste tridimensionnelle lorsque ce n'est pas le cas, l'humidité se propageant dans les trois directions. Si les couches ont un comportement diffusif isotrope, la formulation devient par contre monodimensionnelle.

\section{Application numériques}

Nous nous proposons maintenant de valider par quelques applications numériques ce modèle limite de diffusion de l'humidité dans des plaques minces stratifiées avec diffusion interfaciale entre les plis. Le problème bidimensionnel est résolu par éléments finis pour la discrétisation spatiale et par différences finies pour la discrétisation temporelle dans le cas d'une plaque symétrique de 4 plis. Les résultats sont confrontés à ceux obtenus par résolution du problème hétérogène tridimensionnel $P^{\epsilon}$ qui sert de référence. Avant de présenter cette confrontation, nous décrivons brièvement la formulation variationnelle et la mise en œuvre numérique de ces problèmes évolutifs.

\subsection{Mise en œuvre numérique}

La formulation variationnelle du problème hétérogène (35)-(40) s'écrit :

$$
\begin{gathered}
c^{\epsilon}(t) \in \mathcal{V}=\left\{v \in H^{1}\left(\Omega^{\epsilon}\right), v \in H^{1}\left(\cup \gamma_{i}^{\epsilon}\right), v=c_{a} \text { sur } \Gamma\right\} \\
\frac{\mathrm{d}}{\mathrm{d} t}\left(b\left(c^{\epsilon}(t), v-c^{\epsilon}(t)\right)\right)+a\left(c^{\epsilon}(t), v-c^{\epsilon}(t)\right)=0, \quad \forall v \in \mathcal{V}
\end{gathered}
$$

où les formes bilinéaires sont données par :

$$
\begin{aligned}
b(u, v)= & \int_{\Omega^{\epsilon}} u(x) v(x) \mathrm{d} x, \\
a(u, v)= & \int_{\Omega^{\epsilon}} \boldsymbol{D}^{\epsilon} \nabla u(x) \nabla v(x) \mathrm{d} x \\
& +\int_{\cup \gamma_{i}^{\epsilon}} \epsilon \tilde{D} \nabla_{T} u(x, t) \nabla_{T} v(x) \mathrm{d} \Gamma
\end{aligned}
$$

où l'opérateur gradient surfacique $\nabla_{T}$ est la restriction du gradient sur $\cup \gamma_{i}^{\epsilon}$.

On voit apparaître dans la forme bilinéaire $a(u, v)$ un terme supplémentaire défini sur les interfaces entre les plis dû à la présence des interfaces diffusives. La discrétisation de ce terme se fait simplement en introduisant des éléments finis linéiques d'épaisseur nulle le long des interfaces affectés de la diffusivité $\tilde{D}$, Benhamida et al. [17].

La formulation du problème homogène bidimensionnel posé sur le plan moyen de la plaque est de même type, sans le terme de gradient surfacique. Sa résolution nécessite de calculer au préalable, en utilisant les résultats de la proposition 2 , le tenseur de diffusivité homogénéisé. Ces calculs de moyenne sont explicites.

Les deux résolutions de $\left(P^{\epsilon}\right)$ et $\left(P^{0}\right)$ ont été effectuées ici à l'aide du code de calculs Modulef, Bernadou et al. [26] en adoptant une interpolation de degré 1 pour la discrétisation spatiale et une méthode de différentiation rétrograde d'ordre 1 qui présente une stabilité inconditionnelle pour la discrétisation temporelle. 


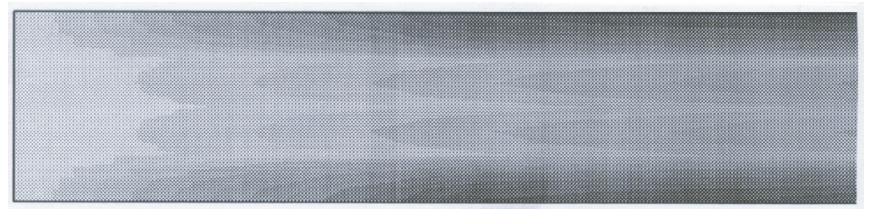

Fig. 1. Isovaleurs de concentration en eau dans la plaque tridimensionnelle.

\subsection{Résultats}

Les résultats que nous présentons ici concernent des plaques stratifiées croisées symétriques $(\theta,-\theta)_{\mathrm{S}}$ composées de quatre couches d'un même pli unidirectionnel, les fibres d'un pli étant orientées respectivement selon l'angle $\theta$ mesuré par rapport à la direction $\boldsymbol{e}_{1}$. Les plaques ont pour dimensions transversales $100 \mathrm{~mm}$ suivant $\boldsymbol{e}_{1}, 50 \mathrm{~mm}$ suivant $\boldsymbol{e}_{2}$ et $2 \mathrm{~mm}$ d'épaisseur, chacun des plis étant de $0,5 \mathrm{~mm}$ d'épaisseur. La concentration $c_{\mathrm{a}}$ sur les faces d'équation $x_{1}=0$. et $x_{1}=100$. est imposée à $1 \mathrm{~g} . \mathrm{mm}^{-3}$, ces faces étant directement en contact avec l'eau.

Les plis unidirectionnels sont réalisés en matrice époxy isotrope de coefficient de diffusion $D_{m}=$ $1,4410^{6} \mathrm{~mm}^{2} \cdot \mathrm{s}^{-1}$, renforcée par des fibres de verre, le taux de renforts étant de $60 \%$. Les fibres de verre sont considérées comme non-absorbantes en s'appuyant sur les constatations expérimentales, P. Bonniau et A.R. Bunsel [10], B. Dewimille [2], B. Dewimille et A.R Bunsell [11]. Le comportement hygrométrique homogène équivalent du pli orienté à $0^{0}$, a été obtenu ici par une approche d'homogénéisation des milieux périodiques, $\mathrm{N}$. Lebris [16], et est donné par :

$$
\boldsymbol{D}_{\mathrm{p}}=D_{\mathrm{m}}\left(\begin{array}{ccc}
0,5 & 0 & 0 \\
0 & 0,335 & 0 \\
0 & 0 & 0,335
\end{array}\right)
$$

La valeur retenue pour le coefficient de diffusion interfaciale $\tilde{D}$ entre les différents plis est de $\tilde{D}=1,44 \times$ $10^{9} \mathrm{~mm}^{2} \cdot \mathrm{s}^{-1}$ et correspond au comportement limite d'une interphase d'épaisseur $e=10^{-3} \mathrm{~mm}$ et de diffusivité $D=10^{3} D_{\mathrm{m}}$. Ces valeurs ont été adoptées en s'appuyant sur des confrontations expérimentales menées sur des unidirectionnels, N. Lebris [16], P. Kanouté [12].

Les isovaleurs de concentration en eau dans la plaque hétérogène tridimensionnelle obtenues dans le cas d'une orientation $\theta=45^{\circ}$ sont présentées à la figure 1 suivant une coupe transversale sur la demi-longueur de la plaque au cours du processus d'absorption. On y observe la propagation préférentielle de l'eau suivant les interfaces entre les plis simulée par la loi d'interface établie à la proposition 1.

Ce calcul évolutif tridimensionnel peut être avantageusement remplacé par une résolution bidimensionnelle posée sur le plan moyen de la plaque en suivant la proposition 2. Le tenseur de diffusité homogène équivalent de la plaque stratifiée avec $\theta=45^{\circ}$, calculé avec la formule (42) est alors :

$$
\boldsymbol{D}^{\mathrm{h}}=D_{\mathrm{m}}\left(\begin{array}{cc}
1,916 & 0 \\
0 & 1,916
\end{array}\right)
$$

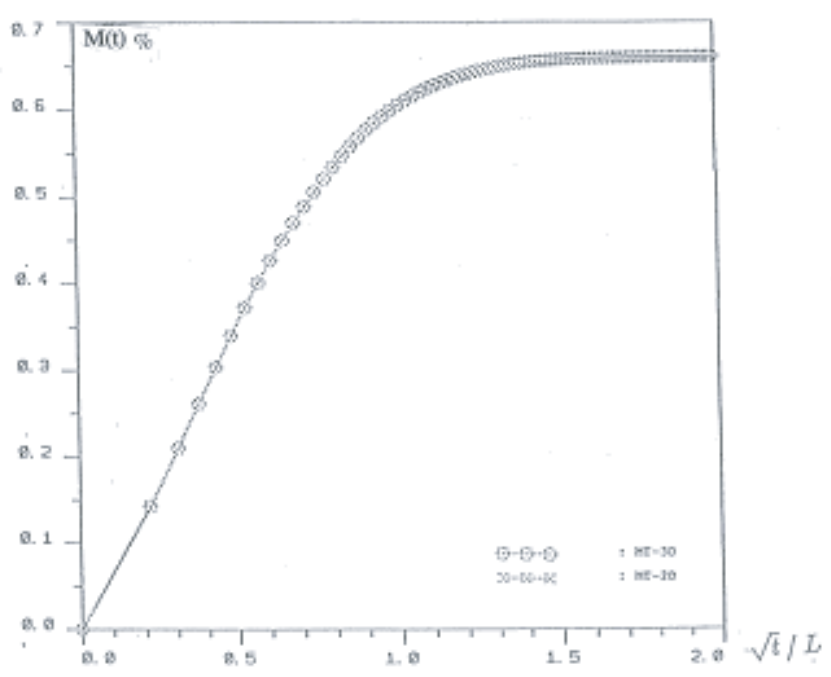

Fig. 2. Évolution temporelle du gain relatif en poids en eau de la plaque. Confrontation du modèle bidimensionnel équivalent $($ symbole $\times$ ) et de l'approche tridimensionnelle (symbole o).

On retrouve bien un comportement diffusif isotrope, classique des empilements croisés $\left(45^{\circ},-45^{\circ}\right)_{\mathrm{S}}$.

Les résultats obtenus par la résolution bidimensionnelle sont confrontés à ceux fournis par le calcul de référence tridimensionnel à travers la figure 2 qui représente l'évolution temporelle du gain relatif en poids en eau $M(t)$ de la plaque en fonction du paramètre $\sqrt{t} / L$, où $L$ désigne la demi-longueur de la plaque selon la direction de diffusion $\boldsymbol{e}_{1}$. Ce gain en poids en eau est calculé dans chacune des deux approches par rapport à la masse sèche de la plaque avec la formule :

$$
M(t)=\frac{1}{\rho_{\mathrm{s}} V} \int_{V} c(x, t) \mathrm{d} x,
$$

où $\rho_{\mathrm{s}}$ désigne la masse volumique de la plaque sèche et $V$ son volume.

Le modèle simplifié de plaque conduit à une très bonne concordance avec le calcul tridimensionnel. La saturation est obtenue pour cette plaque après un temps d'exposition d'environ 39 ans et le gain en poids de la plaque à saturation est de $66 \%$. La plaque étant uniquement exposée à l'eau sur sa face latérale, le processus d'absorption d'humidité est très lent, conformément aux observations expérimentales, P. Kanouté [12].

L'influence des interfaces diffusives sur le comportement des plaques est mise en évidence à la figure 3 par la représentation polaire des coefficients de diffusion équivalents $D_{11}^{\mathrm{h}}$ et $D_{22}^{\mathrm{h}}$ pour une plaque $(\theta,-\theta)_{\mathrm{S}}$ en fonction de l'angle $\theta$. Le calcul du comportement incluant des interfaces diffusives, désigné par (AI), y est comparé à celui avec interfaces non diffusives désigné par (SI) correspondant au cas où $(\tilde{D}=0)$. Naturellement, la présence d'interfaces diffusives conduit à des coefficients de diffusion équivalents de la plaque plus importants. Le caractère anisotrope de la diffusion dans la plaque s'atténue 


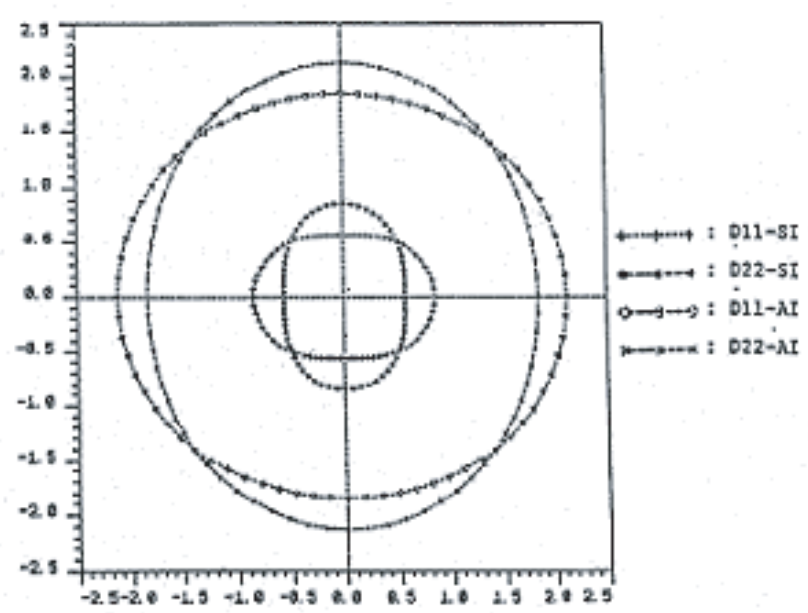

Fig. 3. Représentation polaire des coefficients de diffusion homogènes équivalents dans une plaque stratifiée $(\theta,-\theta)_{\mathrm{S}}$, avec interfaces diffusives (AI) et sans interfaces diffusives (SI).

lorsque l'on prend en compte la diffusivité des interfaces. Ces dernières, qui ont un comportement supposé isotrope, tendent en effet à imposer leur isotropie globalement à la plaque stratifiée.

\section{Conclusion}

La modélisation de la diffusion de l'humidité dans des composites stratifiés suivant une cinétique d'absorption fickienne a été enrichie dans ce travail par l'introduction d'un modèle de diffusion interfaciale. En s'appuyant sur des constatations expérimentales, les intercouches entre les plis du stratifié, qui sont très fines et fortement diffusives, ont été avantageusement remplacées par une loi surfacique asymptotiquement équivalente. Les problèmes numériques liés à la discrétisation de domaines de très faible épaisseur se trouvent ainsi évités. Cette loi, introduite entre les plis d'une plaque stratifiée mince, dont les faces supérieure et inférieure sont isolées, conduit à la formulation d'un modèle de diffusion bidimensionnel. Là encore, sur le plan numérique, cette approche bidimensionnelle apparaît attractive au vu des exemples numériques présentés.

Ce travail pourrait être affiné et complété de façon intéressante par des confrontations à des travaux expérimentaux. L'identification de la diffusion interfaciale devrait pouvoir être menée comme cela a été fait avec succès sur d'autres types de composites. Par ailleurs, les modélisations proposées doivent pouvoir s'étendre à une cinétique d'absorption de Langmuir, qui tiendrait ainsi compte, outre de la diffusion, du caractère piégeant et dépiégeant de l'eau des interfaces. Le couplage des modèles proposés avec les équations de hygroélasticité pourra ensuite permettre d'étudier le phénomène de gonflement des composites immergés.

\section{Références}

[1] G.S. Springer, Moisture content of composites under transient conditions, J. Composite Materials 11 (1977)

[2] B. Dewimille, Hygrothermal ageing of an unidirectionnal glass-fiber Epoxy composite during water immersion, ICCM3 1 (1980) 597

[3] R. Delasi, J.B. Whiteside, Effect of moisture on epoxy resins and composites, Advanced Composite Materials Environnemental effects, ASTM STP 658 (1978) 2-20

[4] C.H. Shen, G.S. Springer, Moisture absorption and desorption of composite materials, Environnemental Effets on Composite Materials (1981) 15-33

[5] A. Loos, G.S. Springer, Moisture absorption of graphite-epoxy immersed in liquids and humid air, Environnemental Effects on Composite Materials (1981) 34-49

[6] G.S. Springer, S.W. Tsai, Thermal conductivities of unidirectional composites, J. Composite Materials 1 (1967) 166-173

[7] J.P. Favre, J.N. Dewas, Diffusion of water in carbon epoxy composite materials. Evaluation of a moded based on matrix absorption, Comptes Rendus des Quatrièmes Journées des Composites, Pluralis Editor, 1984, pp. 631644

[8] J. Crank, The mathematics of diffusion, Oxford Editor, 1967

[9] R. Baizeau, Comportement à long terme de tubes composites sous contraintes en milieu humide, Rapport interne IFREMER, DITI/GO/MM 94-18, (1994)

[10] P. Bonniau, A.R. Bunsell, A comparative study of water absorption theories applied to glass epoxy composite, J. Composite Materials 5 (1981) 272-295

[11] B. Dewimille, A.R. Bunsell, Accelerated ageing of glass fibre-reinforced epoxy resin in water, Composites (1983) $35-40$

[12] P. Kanouté, Étude du comportement hygromécanique de matériaux composites à matrice polymérique, thèse de Doctorat de l'Université P. et M. Curie, 1999

[13] J.P. Favre, Caractéristiques physiques et hygrothermiques des composites, Étude de l'interface, École d'hiver, Piau-Engaly, 1989

[14] C. Schutte, Environmental durability of glass-fiber composites, 11 th Materials Science and Engineering 13 (1994) 265-324

[15] A. Benhamida, H. Dumontet, N. Lebris, A. Lekhder, Rôle des interfaces dans le transport de l'humidité dans des matériaux composites, Deuxième Colloque National en Calcul des Structures, Giens France, 2, 1995, pp. 787-792

[16] N. Lebris, Modélisation du comportement à long terme des matériaux composites : Propagation de l'humidité, Fluage d'enceintes océanographiques, Thèse de Doctorat de l'Université P. et M. Curie, 1999

[17] A. Benhamida, H. Dumontet, A. Lekhder, Un modèle de comportement hygroscopique des mousses syntactiques, Revue des composites et des matériaux avancés 8 (1998) 73-90

[18] A. Lekhder, Diffusion de l'humidité dans des matériaux composites. Influence de la diffusion interfaciale, Thèse d'État de la Faculté des Sciences de Rabat, Maroc, 1999 
[19] H.S. Carslaw, J.C. Jaeger, Conduction of Heat, Oxford Editor, 1959

[20] H.P. Huy et E. Sanchez-Palencia, Phénomènes de transmission à travers des couches minces de conductivité élevée, J. Mathematical Analysis and Applications 47 (1974) 284-309

[21] A. Bendali, Analyse asymptotique de la diffraction d'une onde électromagnétique TH ou TE par un conducteur recouvert d'une couche mince diélectrique, Rapport interne CMAP, École Polytechnique, 1994

[22] Y. Chevalier, Comportements élastique et viscoélastique des composites, Techniques de l'Ingénieur 5 (1988)
[23] P. Destuynder, P.G. Ciarlet, A justification of two dimensional linear plate model, J. Mécanique 18 (1979) 315-344

[24] D. Caillerie, Thin elastic and periodic plates, Mathematical Methods in Applied Sciences 6 (1979) 159-191

[25] E. Sanchez-Palencia, Les méthodes de l'homogénéisation : Théorie et applications en physiques, éditions Masson, 1985

[26] M. Bernadou, P.L. George, A. Hassim, P. Joly, P. Lang, B. Muller, A. Perronnet, E. Saltel, D. Steer, G. Vanderborck, M. Vidrascu, Modulef a modular library of finite elements, Inria, 1988 